hep-ph/0004129

April 2000

\title{
NEW DIRECTIONS FOR NEW DIMENSIONS: FROM STRINGS TO NEUTRINOS TO AXIONS TO... ${ }^{a}$
}

\author{
Keith R. Dienes \\ Theory Division, CERN, CH-1211 Geneva 23, Switzerland \\ Department of Physics, University of Arizona, Tucson, AZ 85721 USA $^{b}$
}

\begin{abstract}
In this talk, I discuss recent developments concerning the possibility of large extra spacetime dimensions. After briefly reviewing how such dimensions can lower the fundamental GUT, Planck, and string scales, I then outline how these scenarios lead to a new higher-dimensional seesaw mechanism for generating neutrino oscillations - perhaps even without neutrino masses. I also discuss how extra dimensions lead to new mechanisms contributing to the "invisibility" of the QCD axion. This talk reports on work done in collaboration with Emilian Dudas and Tony Gherghetta.
\end{abstract}

\section{Introduction: Lowering the fundamental scales of physics}

The possibility of large extra spacetime dimensions has recently received considerable attention. This is clearly an exciting prospect. One of the earliest proponents of $\mathrm{TeV}$-scale extra dimensions was Antoniadis $\mathrm{t}$, who attempted th use such extra dimensions to explain supersymmetry breaking. Later, Witten pointed out that extra large dimensions could lower the string scale below its usual value near $10^{18} \mathrm{GeV}$, and subsequently Lykken 3 proposed that Witten's idea could be extended to lower the string scale all the way to the TeV-range. Finally, in March 1998, it was proposed that extra dimensions could also be used to lower the fundamental Planck scale 6 as well as the fundamental GUT scale 3 . Thus, combining these different proposals, it becomes possible to contemplate a self-consistent scenario in which all high fundamental energy scales (GUT, Planck, and string scales) are eliminated in favor of large extra spacetime dimensions!

It is important to distinguish two different types of extra spacetime dimensions. First, there are so-called "universal" extra dimensions. These extra

\footnotetext{
${ }^{a}$ Invited plenary talk given at PASCOS '99: $7^{\text {th }}$ International Symposium on Particles, Strings, and Cosmology (held at Lake Tahoe, California, 10-16 December 1999). To appear in the Proceedings.

${ }^{b}$ Current address. E-mail: dienes@physics.arizona.edu
} 
dimensions are experienced by all forces, both gauge and gravitational; in technical terminology, these extra dimensions are "in the brane". Because they affect the gauge forces (as probed by accelerator experiments), such dimensions can be no larger than roughly an inverse TeV. By contrast, the second class of extra dimensions are felt only by gravity; they are perpendicular to the D-brane on which the gauge forces are localized, and may therefore be considered "off the brane". The sizes of such extra dimensions are significantly less constrained, and may in fact be as large as a millimeter.

Both of these types of extra dimensions play a role in lowering the fundamental scales of physics. Indeed, as outlined above, there are three different proposals: extra dimensions to lower the GUT scale 6 , extra dimensions to

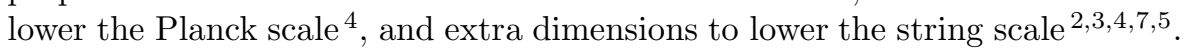
We shall now briefly review these three proposals.

In the proposal of Ref. 1 to lower the GUT scale, one introduces some number $\delta$ of "universal" extra spacetime dimensions "in the brane" [so that the Standard Model resides on a $D(3+\delta)$ brane], and imagines that these dimensions have a common radius $R$. Because these extra dimensions are felt by the gauge forces, they change the running of the three gauge couplings from logarithmic to power-law behavior:

$$
\alpha_{i}^{-1}(\mu)=\alpha_{i}^{-1}\left(\mu_{0}\right)-\frac{b_{i}-\tilde{b}_{i}}{2 \pi} \ln (R \mu)-\frac{\tilde{b}_{i} X_{\delta}}{2 \pi \delta}\left[(R \mu)^{\delta}-1\right] .
$$

The emergence of power-law behavior is expected simply from dimensional analysis, since the gauge couplings themselves become dimensionful in higher dimensions, and hence have a classical scaling in addition to their quantummechanical (logarithmic) running. This power-law behavior can also be realized via a Kaluza-Klein summation, as discussed in Refs.10.6. In Eq. (17), $X_{\delta}$ is a normalization constant and $\left(b_{i}, \tilde{b}_{i}\right)$ represent the one-loop beta-functions appropriate for the zero-mode and excited Kaluza-Klein states respectively. The exact values of these beta-functions depend on details of the compactification,

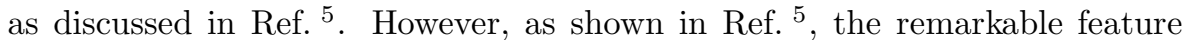
of this higher-dimensional running is that gauge coupling unification is still generally preserved, but with a lowered unification scale! As an interesting case, let us consider $R^{-1}=1 \mathrm{TeV}$ and $\delta=1$. With one-loop beta-function coefficients $\left(b_{1}, b_{2}, b_{3}\right)=(33 / 5,1,-3)$ and $\left(\tilde{b}_{1}, \tilde{b}_{2}, \tilde{b}_{3}\right)=(3 / 5,-3,-\overline{6})$, corresponding to a certain orbifold compactification discussed in Ref. find the unification shown in Fig. 1. An important point to notice is that no large hierarchy is needed between the scale of the extra dimensions and the lowered GUT scale.

This reduced-scale unification leads to many important quantitative ques- 


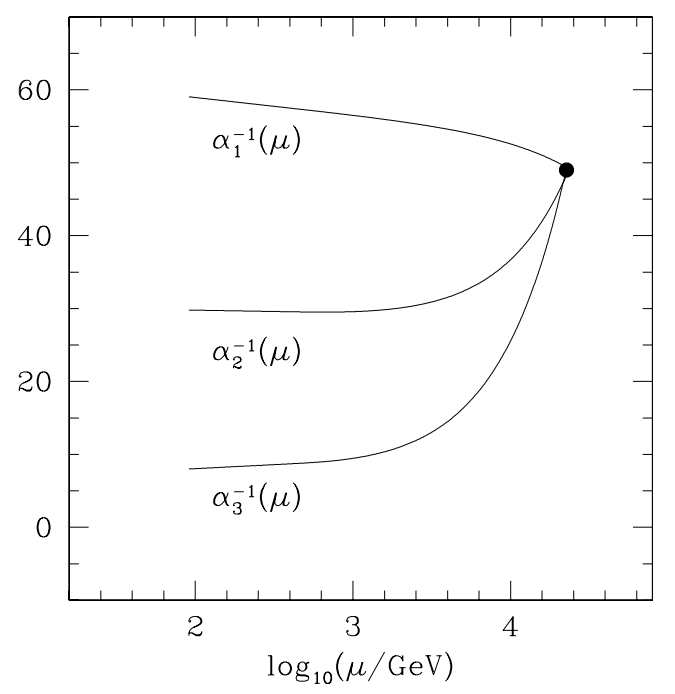

Figure 1: Unification of gauge couplings at the new unification scale $M_{\mathrm{GUT}}^{\prime} \approx 20 \mathrm{TeV}$, assuming the appearance of a single extra spacetime dimension of radius $R^{-1}=1 \mathrm{TeV}$.

tions. How predictive is this unification? How perturbative is it? How sensitive is it to unification-scale effects? What about higher-loop corrections? These issues are discussed in Ref.6. The upshot is that this sort of unification scenario is predictive, perturbative, and not unreasonably sensitive to unification-scale effects. There have also been many further extensions of these basic ideas 6 . These include the study of two- and higher-loop effects; the incorporation of extra matter beyond the MSSM in order to increase the numerical accuracy of the unification; alternative derivations of these RGE's from a Wilsonian perspective; studies of regularization independence; the extension of these ideas to the power-law running of Yukawa couplings; the higher-dimensional evolution of soft supersymmetry-breaking mass parameters; multi-step higher-dimensional unification scenarios; and alternative embeddings of the Standard Model into higher dimensions. Alternative ideas pertaining to reduced-scale gauge unification have also been discussed in Refs. 6 .

Extra dimensions can also be used to lower the Planck scale, as pointed out in Ref. 6 . Indeed, in many respects this Planck-scale proposal and the above GUT-scale proposal are the gravitational/gauge counterparts of each other. Whereas the GUT proposal utilizes $\delta$ extra dimensions "in the brane" with ra- 
dius $R$ to modify the running of the three gauge couplings, the Planck proposal of Ref. 4 utilizes some number $n$ of extra dimensions of radius $r$ "off the brane" to modify the running of the effective dimensionless gravitational (Newton) coupling $\tilde{G}_{N}(\mu) \equiv \mu^{2} G_{N}$. As expected, the presence of the extra dimensions enhances the power-law running of this gravitational coupling, changing the scaling behavior from $\mu^{2}$ to $\mu^{2+n}$. This in turn lowers the Planck scale [i.e., the fundamental gravitational scale, defined as the scale where $\left.\tilde{G}_{N}(\mu) \sim \mathcal{O}(1)\right]$. Unlike the GUT proposal, however, one typically requires a significant hierarchy between the scale of the extra dimensions and the lowered Planck scale. For example, in the case $n=2$ with a lowered Planck scale in the TeV-range, one finds $r \approx$ millimeter $\approx\left(10^{-4} \mathrm{eV}\right)^{-1}$. This hierarchy is exactly as large as the original hierarchy between the electroweak scale and the usual fourdimensional Planck scale. []

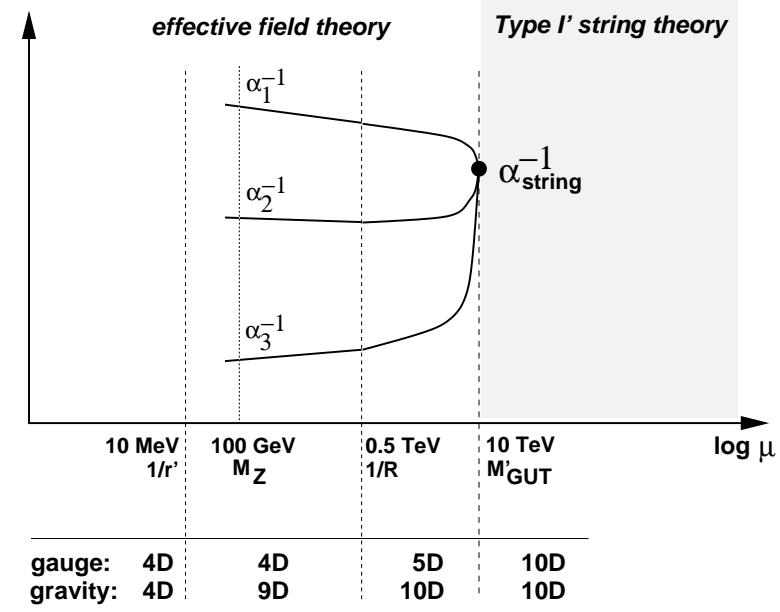

Figure 2: The unification of gauge and gravitational couplings within the framework of a Type I string theory, assuming a string scale at $M_{\text {string }}=10 \mathrm{TeV}$.

Finally, combinations of both types of extra dimensions can be used to lower the string scale 6 . For Type I strings, the string scale ultimately depends on the six-volume $V_{6}$ of compactification from ten flat dimensions to four flat

${ }^{a}$ As pointed out in Ref.11, it may be possible to avoid the former hierarchy and nevertheless explain the latter hierarchy by virtue of a "warp" rescalingfactor. Issues surrounding gauge coupling unification in this scenario are discussed in Ref. 12. 
dimensions:

$$
M_{\text {string }} \sim \sqrt{\frac{1}{\alpha_{\mathrm{GUT}} M_{\text {Planck }}}} V_{6}^{-1 / 4} .
$$

Therefore, as discussed in Ref. 5 , if we seek to combine the above GUT and Planck scenarios together within string theory, we can write $V_{6}=R^{\delta} r^{6-\delta}$ where $(R, \delta)$ describe the extra dimensions "in" the brane (to produce a lowered GUT scale) and $(r, 6-\delta)$ describe the extra dimensions "off" the brane (to produce a lowered Planck scale). If we demand that the lowered GUT scale coincide with $M_{\text {string}}$, one can then solve to obtain a self-consistent solution. For example, let us consider the case with $\delta=1$ and $R^{-1} \approx 0.5 \mathrm{TeV}$. (This extreme value may already be ruled out experimentally, but will serve our illustrative purposes.) This implies that $M_{\text {GUT }}^{\prime} \approx 10 \mathrm{TeV}$, which in turn implies (after a T-duality transformation) that the remaining five extra dimensions must have radius $r \approx(10 \mathrm{MeV})^{-1}$.

Thus, putting the pieces together in this example, we are led to a unified embedding into string theory, as illustrated in Fig. 2. Above the string scale $M_{\text {string }}=10 \mathrm{TeV}$, the physics is described in terms of a full Type I string theory. Below $10 \mathrm{TeV}$, by contrast, the physics is described by a series of effective field theories in which the gauge and gravitational forces feel different numbers of spacetime dimensions. Together, everything is balanced so as to produce a self-consistent simultaneous lowering of GUT, Planck, and string scales. Of course, other configurations are also possible.

\section{Light neutrino masses without heavy mass scales: A higher-dimensional seesaw mechanism}

As we have seen, the lesson from the above developments has been that heavy mass scales in four dimensions can be replaced by lighter mass scales in higher dimensions. However, low-energy neutrino data seem to provide independent evidence for yet another heavy mass scale, namely the seesaw scale. The seesaw mechanism relies on the existence of a new heavy mass scale $M \approx$ $M_{\mathrm{GUT}}$ associated with a right-handed neutrino singlet field $N$. The question then emerges whether it is possible to generate light neutrino masses without the introduction of a heavy mass scale, perhaps by some intrinsically higherdimensional mechanism.

To date, there have been essentially two ideas concerning how this might be accomplished within the large extra-dimension framework: one proposal 13 utilizes a higher-dimensional seesaw mechanism, and the other 14 utilizes a higherdimensional volume factor. Both proposals originate with the same observation: because the right-handed neutrino is a Standard-Model gauge singlet, it 
need not be restricted to a "brane" with respect to the full higher-dimensional space. It is therefore possible for this field to experience extra spacetime dimensions and thereby accrue an infinite tower of Kaluza-Klein excitations. This then leads to a number of higher-dimensional mechanisms 1324 . 15 for suppressing the resulting neutrino mass without a heavy mass scale. In the following, we shall concentrate on one of the mechanisms advanced in Ref.13, namely the intriguing possibility that in higher dimensions, neutrino oscillations need not imply the existence of neutrino masses at all! This would then eliminate the need for a high fundamental scale. Other mechanisms for explaining light but non-zero neutrino masses are also discussed in Refs. $13,14,15$.

We begin by assuming that the right-handed neutrino feels extra dimensions, while the left-handed neutrino $\nu_{L}$ does not. For concreteness, we consider a Dirac fermion $\Psi$ in five dimensions, and work in the Weyl basis in which $\Psi$ can be decomposed into two two-component spinors: $\Psi=\left(\psi_{1}, \bar{\psi}_{2}\right)^{T}$. When the extra spacetime dimension is compactified on a $\mathbb{Z}_{2}$ orbifold, it is natural for one of the two-component Weyl spinors, e.g., $\psi_{1}$, to be taken to be even under the $\mathbb{Z}_{2}$ action $y \rightarrow-y$, while the other spinor $\psi_{2}$ is taken to be odd. If the left-handed neutrino $\nu_{L}$ is restricted to a brane located at the orbifold fixed point $y=0$, then $\psi_{2}$ vanishes at this point and so the most natural coupling is between $\nu_{L}$ and $\psi_{1}$. For generality, we will also include a possible "bare" Majorana mass term for $\Psi$ of the form $\frac{1}{2} M_{0} \bar{\Psi}^{\mathrm{c}} \Psi$. This then results in a Lagrangian of the form

$$
\begin{aligned}
\mathcal{L}= & \int d^{4} x d y M_{s}\left\{\bar{\psi}_{1} i \bar{\sigma}^{\mu} \partial_{\mu} \psi_{1}+\bar{\psi}_{2} i \bar{\sigma}^{\mu} \partial_{\mu} \psi_{2}+\frac{1}{2} M_{0}\left(\psi_{1} \psi_{1}+\psi_{2} \psi_{2}+\text { h.c. }\right)\right\} \\
& +\int d^{4} x\left\{\bar{\nu}_{L} i \bar{\sigma}^{\mu} D_{\mu} \nu_{L}+\left(\left.\hat{m} \nu_{L} \psi_{1}\right|_{y=0}+\text { h.c. }\right)\right\}
\end{aligned}
$$

where $y$ is the coordinate of the extra compactified spacetime dimension and where $M_{s}$ is the mass scale of the higher-dimensional fundamental theory (e.g., a reduced Type I string scale). Note that the last term represents the Dirac brane/bulk Yukawa coupling between $\nu_{L}$ and $\psi_{1}$.

Next, we compactify the Lagrangian (3) down to four dimensions by expanding the five-dimensional $\Psi$ field in Kaluza-Klein modes. Imposing the orbifold relations $\psi_{1,2}(-y)= \pm \psi_{1,2}(y)$ implies that our Kaluza-Klein decomposition takes the form $\psi_{1}(x, y)=(2 \pi R)^{-1 / 2} \sum_{n=0}^{\infty} \psi_{1}^{(n)}(x) \cos (n y / R)$ and a similar result for $\psi_{2}$ with cosine replaced by sine. For convenience, we shall also define the linear combinations $N^{(n)} \equiv\left(\psi_{1}^{(n)}+\psi_{2}^{(n)}\right) / \sqrt{2}$ and $M^{(n)} \equiv$ $\left(\psi_{1}^{(n)}-\psi_{2}^{(n)}\right) / \sqrt{2}$ for all $n>0$. Inserting this decomposition into Eq. (3) and integrating over the compactified dimension, we then obtain an effective four- 
dimensional Lagrangian in which the Standard-Model neutrino $\nu_{L}$ mixes with the entire tower of Kaluza-Klein states of the higher-dimensional $\Psi$ field with a mass mixing matrix of the form

$$
\mathcal{M}=\left(\begin{array}{ccccccc}
0 & m & m & m & m & m & \ldots \\
m & M_{0} & 0 & 0 & 0 & 0 & \ldots \\
m & 0 & M_{0}+1 / R & 0 & 0 & 0 & \ldots \\
m & 0 & 0 & M_{0}-1 / R & 0 & 0 & \ldots \\
m & 0 & 0 & 0 & M_{0}+2 / R & 0 & \ldots \\
m & 0 & 0 & 0 & 0 & M_{0}-2 / R & \ldots \\
\vdots & \vdots & \vdots & \vdots & \vdots & \vdots & \ddots
\end{array}\right) .
$$

In Eq. (雨), we have defined the basis $\left(\nu_{L}, \psi_{1}^{(0)}, N^{(1)}, M^{(1)}, N^{(2)}, M^{(2)}, \ldots\right)$. Note that $m \equiv \hat{m} / \sqrt{2 \pi R M_{s}}$ is the Dirac coupling suppressed by a volume factor corresponding to the extra spacetime dimension.

While in principle any value for $M_{0}$ is allowed (depending on the structure of the full effective Lagrangian derived from the particular underlying string model), the topological constraints associated with Scherk-Schwarz compactification naturally suggest two specific values: $M_{0}=0$ (corresponding to no breaking of lepton number), and $M_{0}=(2 R)^{-1}$ (corresponding to a global breaking of lepton number). Let us here consider the non-trivial possibility $M_{0}=(2 R)^{-1}$. Note that this value is fixed topologically, and hence does not require any fine-tuning. It is then possible to solve for the eigenvalues and eigenstates of the mass mixing matrix in Eq. (四). Remarkably, it turns out that for any value of $m R$, there exists an exactly zero eigenvalue, with a corresponding mass eigenstate given exactly by

$$
\left|\tilde{\nu}_{L}\right\rangle=\frac{1}{\sqrt{1+\pi^{2} m^{2} R^{2}}}\left\{\left|\nu_{L}\right\rangle-m R \sum_{k=1}^{\infty} \frac{1}{k-1 / 2}\left[\left|N^{(k-1)}\right\rangle-\left|M^{(k)}\right\rangle\right]\right\}
$$

where we have defined $N^{(0)} \equiv \psi_{1}^{(0)}$. Eyen though this result is exact for all $m R$, in most realistic scenarios (see Ref. 13 ), we have $m R \ll 1$. Thus, we see that even though this neutrino mass eigenstate contains a small, non-trivial admixture of Kaluza-Klein states, the dominant component of our massless neutrino eigenstate remains the left-handed gauge-eigenstate neutrino $\nu_{L}$, as required phenomenologically. Nevertheless, this particular admixture of excited KaluzaKlein states has rendered the neutrino eigenstate exactly massless! In other words, the effects of the infinite tower of Kaluza-Klein states for the $\Psi$ field have driven the neutrino mass exactly to zero.

One might still worry that a vanishing neutrino mass is unacceptable be- 
cause of the recent evidence for neutrino oscillations. However, even though the neutrino mass is vanishing in this scenario, there continue to exist oscillations because of the non-trivial mixings between the left-handed neutrino and the infinite tower of Kaluza-Klein states. Specifically, upon diagonalizing the mass matrix (1) and calculating the resulting oscillation probabilities in the usual way, we find 13 the result shown in Fig. 3. This figure thus provides explicit verification that neutrino oscillations do indeed occur, even though the physical neutrino is exactly massless. Of course, we have been discussing only the simple case of neutrino/anti-neutrino oscillations. However, this mechanism can easily be generalized to include the case of flavor oscillations as well.

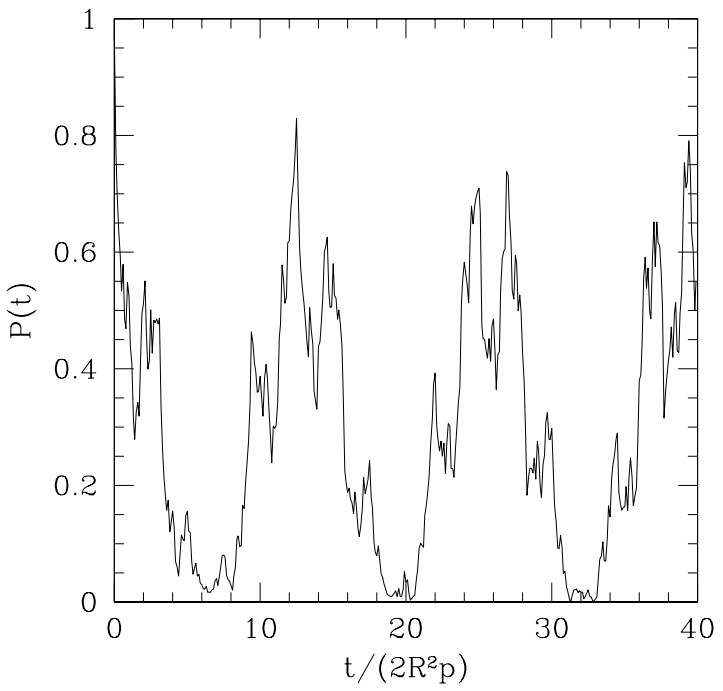

Figure 3: Higher-dimensional neutrino/anti-neutrino oscillations, even when the neutrino itself is massless. Here we have plotted the probability that the gauge neutrino eigenstate $\nu_{L}$ is preserved as a function of time. The multi-component nature of the mixing between $\nu_{L}$ and the infinite set of right-handed Kaluza-Klein neutrinos is reflected in the jagged shape of the oscillations. Note that the neutrino deficitare total even though the neutrino regenerations are only partial. See Ref. 13 for further details.

At first sight, it may seem strange that we are able to have neutrino oscillations without neutrino masses. However, the crucial point is that once the infinite towers of Kaluza-Klein states are included, the mixing mass matrix (画) with $M_{0}=(2 R)^{-1}$ yields a zero eigenvalue without becoming diagonal. This is not possible in the usual four-dimensional neutrino/anti-neutrino scenarios, 
where the analogous mixing matrix takes the simple form $\left(\begin{array}{cc}0 & m \\ m & M_{0}\end{array}\right)$. Indeed, the masses of the right-handed Kaluza-Klein states themselves are sufficient to generate the desired oscillations indirectly, even though these Kaluza-Klein states are in the "bulk" rather than on the brane. Thus, if such a scenario can be realized within the context of a fully realistic string model, then the recent observations of neutrino oscillations can be re-interpreted not as providing evidence for neutrino masses, but rather as providing evidence for extra spacetime dimensions!

\section{Extra dimensions and "invisible" axions}

Many of the above ideas are completely general, and apply to other bulk fields as well. Towards this end, let us now discuss how extra spacetime dimensions may contribute to the invisibility of the QCD axion. Like the graviton and right-handed neutrino, the QCD axion is also a Standard-Model singlet. The QCD axion is therefore free to propagate into the bulk.

Can this be used to lower the fundamental Peccei-Quinn (PQ) symmetrybreaking scale? This issue has been investigated in Refs. 16,17. As explicitly shown in Ref. 17 (and first proposed in Ref.4), it is indeed possible to exploit the volume factor of large extra dimensions in order to realize a large effective four-dimensional PQ scale from a smaller, higher-dimensional fundamental PQ scale. Thus, once again, no large fundamental energy scales are required.

However, as discussed in Ref.17, the presence of Kaluza-Klein axions can have important and unexpected effects on axion phenomenology. Just as in the neutrino case discussed above, the naïve four-dimensional axion mixes with the infinite tower of Kaluza-Klein axions, with a mass matrix given in Ref.17. This mixing has a number of interesting phenomenological consequences. First, as shown in Ref.17, under certain circumstances the mass of the axion essentially decouples from the $\mathrm{PQ}$ scale, and instead is set by the radius of the extra spacetime dimension! Thus, axions in the $10^{-4} \mathrm{eV}$ mass range are consistent with (sub-)millimeter extra dimensions. This decoupling implies that it may be possible to adjust the mass of the axion independently of its couplings to matter. This is not possible in four dimensions.

Second, as discussed in Ref. 17, the usual four-dimensional axion is no longer a mass eigenstate because of the non-trivial axion mass mixing matrix. This implies that the four-dimensional axion should undergo laboratory oscillations which are entirely analogous to neutrino oscillations. Moreover, because the axion is now a bulk field, Standard-Model particles couple not only to the axion zero-mode, but rather to the entire linear superposition 
$a^{\prime} \sim \sum_{n} a_{n}$ (where $a_{n}$ are the axion Kaluza-Klein modes). Therefore, the quantity of phenomenological interest is the probability $P_{a^{\prime} \rightarrow a^{\prime}}(t)$ that $a^{\prime}$ is preserved as a function of time. This probability 17 is shown in Fig. 1 . Unlike the neutrino case, we see that the probability drops rapidly from 1 (at the initial time $t=0$ ) to extremely small values (expected to be $\approx 10^{-16}$ when an appropriately truncated set of $10^{16}$ Kaluza-Klein states are included in $\left.a^{\prime}\right)$. At no future time does this probability regenerate. Essentially, the axion state $a^{\prime}$ has "decohered" and becomes invisible with respect to subsequent laboratory interactions. This decoherence is therefore a possible mechanism contributing to an invisible axion.

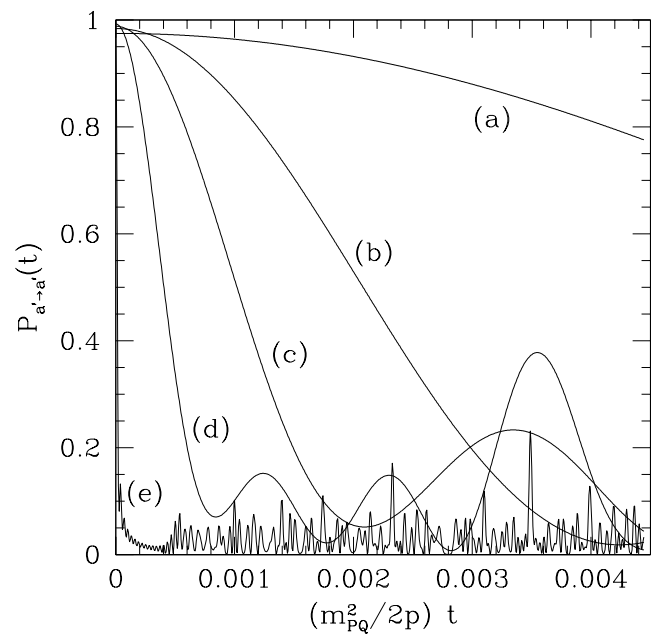

Figure 4: The axion preservation probability $P_{a^{\prime} \rightarrow a^{\prime}}(t)$ as a function of the number $n_{\max }$ of axion Kaluza-Klein states which are included in the analysis. In this plot we show the results for (a) $n_{\max }=1$; (b) $n_{\max }=2$; (c) $n_{\max }=3$; (d) $n_{\max }=5$; and (e) $n_{\max }=30$. As $n_{\max }$ increases, the axion probability rapidly falls to zero as a result of the destructive interference of the Kaluza-Klein states, and remains suppressed without significant axion regeneration at any later times. This "decoherence" of the axion implies that there is negligible probability for subsequently detecting the original axion state atany future time. Further details can be found in Ref.t].

Finally, one can investigate the effects of Kaluza-Klein axions on cosmological relic axion oscillations. In this regard it is important to understand whether the coupled axion Kaluza-Klein states accelerate or retard the dissipation of the cosmological energy density associated with these oscillations. Remarkably, one finds 17 that the net effect of these coupled Kaluza-Klein ax- 
ions is to either preserve or enhance the rate of energy dissipation. This implies that the usual relic oscillation bounds are loosened in higher dimensions, which suggests that it may be possible to raise the effective PQ symmetry-breaking scale beyond its usual four-dimensional value. This could therefore potentially serve as another factor contributing to axion invisibility. Together, these results suggest that it may be possible to develop a new, higher-dimensional approach to axion phenomenology.

\section{Conclusions}

Only experiment will decide if large extra spacetime dimensions actually exist, and if the fundamental high-energy scales of physics are really as low as the TeV-range. Nevertheless, what is remarkable about the recent developments is that they illustrate that the fundamental energy scales are not immutable, and that the parameter space for physics beyond the Standard Model is significantly broader than had been previously thought. Moreover, it is equally remarkable and gratifying that ideas originally born in string theory are having such a profound effect on the answers to primarily phenomenological questions, and that these ideas may be potentially testable in the not-too-distant future. If nothing else, these may be the most valuable lessons that we may take with us from the brane world.

\section{Acknowledgments}

I wish to thank the organizers of the PASCOS '99 conference, and especially Jack Gunion, for the opportunity to speak at such a stimulating and wideranging conference.

\section{References}

1. I. Antoniadis, Phys. Lett. B246 (1990) 377.

2. E. Witten, Nucl. Phys. B471 (1996) 135.

3. J.D. Lykken, Phys. Rev. D54 (1996) 3693.

4. N. Arkani-Hamed, S. Dimopoulos, and G. Dvali, hep-ph/9803315; hep-ph/9807344; I. Antoniadis et al., hep-ph/9804398.

5. K.R. Dienes, E. Dudas, and T. Gherghetta, Phys. Lett. B436 (1998) 55 hep-ph/9803466; Nucl. Phys. B537 (1999) 47 hep-ph/9806292].

6. K.R. Dienes, E. Dudas, and T. Gherghetta, hep-ph/9807522.

7. G. Shiu and S.-H.H. Tye, hep-th/9805157; Z. Kakushadze and S.H.H. Tye, hep-th/9809147. 
8. See, e.g., D. Ghilencea and G.G. Ross, hep-ph/9809217; S.A. Abel and S.F. King, hep-ph/9809467; Z. Kakushadze, hep-th/9811193; Z. Kakushadze and T. Taylor, hep-th/9905137; I. Antoniadis et al., hep-ph/9810410; T. Kobayashi et al., hep-ph/9812221; A. Delgado, A. Pomarol, and M. Quirós, hep-ph/9812489; P. Nath and M. Yamaguchi, hep-ph/9902323, hep-ph/9903298; P. Nath, Y. Yamada, and M. Yamaguchi, hep-ph/9905415; C.D. Carone, hep-ph/9902407, hep-ph/9907362; A. Delgado and M. Quiros, hep-ph/9903400; P.H. Frampton and A. Rasin, hep-ph/9903479; J.W. Moffat, hep-ph/9904446; A. Perez-Lorenzana and R.N. Mohapatra, hep-ph/9904504; H.-C. Cheng, B.A. Dobrescu, and C.T. Hill, hep-ph/9906327; D. Dumitru and S. Nandi, hep-ph/9906514; J.D. Lykken and S. Nandi, hep-ph/9908505; E.G. Floratos and G.K. Leontaris, hep-ph/9906238; K. Huitu and T. Kobayashi, hep-ph/9906431; G.K. Leontaris and N.D. Tracas, hep-ph/9908462; J. Kubo, H. Terao, and G. Zoupanos, hep-ph/9910277; A. Dedes and P. Kanti, hep-ph/0003051.

9. See, e.g., C. Bachas, hep-ph/9807415; K. Benakli, hep-ph/9809582; C.P. Burgess, L.E. Ibáñez, and F. Quevedo, hep-ph/9810535; L.E. Ibáñez, C. Muñoz, and S. Rigolin, hep-ph/9812397; L.E. Ibáñez, hep-ph/9905311; E. Halyo, hep-ph/9905577; N. Arkani-Hamed et al., hep-ph/9908146.

10. T.R. Taylor and G. Veneziano, Phys. Lett. B212 (1988) 147.

11. L. Randall and R. Sundrum, hep-th/9906064.

12. K.R. Dienes, E. Dudas, and T. Gherghetta, Nucl. Phys. B567 (2000) 111 hep-ph/9908530.

13. K.R. Dienes, E. Dudas, and T. Gherghetta, Nucl. Phys. B557 (1999) 25 hep-ph/9811428.

14. N. Arkani-Hamed et al., hep-ph/9811448.

15. See, e.g., G. Dvali and A. Smirnov, hep-ph/9904211; A. Pilaftsis, hep-ph/9906228; A. Das and O. Kong, hep-ph/9907272; R.N. Mohapatra, S. Nandi, and A. Perez-Lorenzana, hep-ph/9907520; A. Ioannisian and A. Pilaftsis, hep-ph/9907522; G.C. McLaughlin and J.N. Ng, hep-ph/9909558, hucl-th/0003023; R.N. Mohapatra and A. Perez-Lorenzana, hep-ph/9910474; A. Ioannisian and J.W.F. Valle, hep-ph/9911349; R. Barbieri, P. Criminelli, and A. Strumia, hep-ph/0002199.

16. S. Chang, S. Tazawa, and M. Yamaguchi, hep-ph/9908515; hep-ph/9909240.

17. K.R. Dienes, E. Dudas, and T. Gherghetta, hep-ph/9912455. 